\title{
IV. Damping of the oscillations in the discharge of a leyden-jar
}

\section{H. Brooks B.A.}

To cite this article: H. Brooks B.A. (1901) IV. Damping of the oscillations in the discharge of a leyden-jar, Philosophical Magazine Series 6, 2:7, 92-108, DOI: $10.1080 / 14786440109462667$

To link to this article: http://dx.doi.org/10.1080/14786440109462667

曲 Published online: 08 Jun 2010.

Submit your article to this journal $\asymp$

Џ Article views: 3

Q View related articles $\longleftarrow$

Citing articles: 5 View citing articles 5 
at the time $\tau$ is further directly proportional to the surface of contact of the reacting parts at the time $\tau_{1}+$ the instability constant $K$. At a constant surface of contact of the reacting parts of the heterogeneous system we have $\Sigma+K=$ constant, $i$. $e$. the instability constant does not become evident from the form of the equation.

Davy-Faraday Laboratory

of the Royal Institution.

IV. Damping of the Oscillations in the Discharge of a Leyden-jar. By H. Brooks, B.A., Tutor in Mathematics, Royal Victoria College for Women, Montreal *

THE method employed in the investigation of this subject depends on the partial demagnetization of a magnetized steel needle when placed inside a solenoid through which a leyden-jar discharge is passed.

The action of a rapidly alternating current on a magnetized steel needle has been investigated by Prof. Rutherford (Trans. Roy. Soc., June 1896), who has shown that the method can be employed as a simple means of comparing the intensities of high-frequency currents, and also as a means of determining the damping.

Erskine (Wied. Ann. vol. Ixii. Oct. 1897) has employed such magnetized steel needles for measurements of the resistance of metals and electrolytes for rapidly alternating currents, and also for the determination of specific inductive capacities.

The object of this investigation was to examine in detail the damping of the electrical oscillations in leyden-jar circuits under varying conditions of spark-length, capacity, and pressure.

The appearance of an air-break in the circuit connecting the outer and inner coatings of the jar when the discharge was passing, was examined by Feddersen by means of a rotating mirror in which the spark was reflected. He found that the image consisted of a series of bright and dark bands rapidly decreasing in breadth and intensity; and when a large resistance was placed in the circuit the image became a broad band of light gradually fading away in intensity.

This method was used by Trowbridge (Phil. Mag. vol. xxx. 1890, and vol. xliii. 1897), who photographed the image of the electric spark drawn out by the rotating mirror and measured the distances between the successive oscillations shown by the dark bands on the photograph. In this way he was enabled to make a comparison of the damping in a few

* Communicated by Prof. E. Rutherford. 
cases; but so complicated are the arrangements for these photographic experiments that no great range of observations could be made; and this is very necessary in order to make any reliable generalizations from so variable a phenomenon as the spark under consideration*.

If two small oppositely-wound solenoids $A$ and $B$ are placed in the discharge-circuit of a leyden-jar and two exactly similar steel needles, both magnetized to suturation, be placed one in each, with their north poles facing in the same direction, then on the passage of a discharge it will be found that the reduction of the magnetic moment is not the same in the two cases. Let $\alpha_{1}, 2, \alpha_{3}$, \&c., be the half-oscillations of the discharge in one direction, and $\beta_{1}, \beta_{2}, \beta_{3}$, \&e., the halfoscillations in the other direction. Suppose that $\alpha_{1}$ tends to magnetize the needle in A still further, no effect is produced, since it is magnetized to saturation, $\beta_{1}$ demagnetizes the surface-layer, $\alpha_{2}$ tends to remove the effects of $\beta_{1}$, and so on. In the solenoid $\mathrm{B}, \alpha_{1}$ demagnetizes the needle, $\beta_{1}$ tends to remagnetize it, $\alpha_{2}$ to undo the effects of $\beta$, and so on. Since the maximum value of the current $\alpha_{1}$ is greater than that of $\beta_{1}$, the needle in $B$ will be more demagnetized than that in $\mathrm{A}$. If, however, the number of turns per centimetre on the solenoid $A$ is increased until the effects on the two needles are exactly the same : then, assuming that the value of the current decreases in geometrical progression, the maximum value of the magnetic force due to the oscillation $\beta_{1}$ acting on $\mathrm{A}$ is equal to the maximum value due to $\alpha_{1}$ acting on $B$. Then if $\gamma_{1}, \gamma_{2}$ are the maximum values of the first and second half-oscillations respectively, and $n_{1}$ and $n_{2}$ the number of turns on $A$ and $B$; then, since

$$
\begin{aligned}
4 \pi n_{1} \gamma_{2} & =4 \pi n_{2} \gamma_{1}, \\
\therefore \quad \frac{\gamma_{2}}{\gamma_{1}} & =\frac{n_{2}}{n_{1}} .
\end{aligned}
$$

The ratio of the second to the first half-oscillation is therefore known, and the damping determined.

The method of two solenoids was not adopted in practice; but one theoretically equivalent was employed, in which it

* Since finishing this investigation my attention has been drawn to a paper by Cardacci (Physikalische Zeitschrift, i. p. 23) on the resistance of the electric spark. The value there given for the ohmic resistance of the spark corresponds very closely, as nearly as I can judge by the data given, with that which I bave obtained; but his results show an increase of resistance with decrease of pressure in air; while all my results in air, hydrogen, and carbonic acid gas show a decrease of resistance with decrease of pressure. 
was necessary to use only one detector-needle. The accuracy of the experiments depends to a rery great extent upon the nature of this neadle. Prof. Rutherford (Trans. Roy. Soc. 1896) has shown that the effect on a magnetized steel needle differs in any given circuit according to the length of the needle, the hardness of the steel, and the thickness of the wire used in its construction. An examination of a needle after it has been partially demagnetized by a discharge, shows a surface-layer magnetized in a direction opposite to the internal magnetization. Since a leyden-jar in general gives several complete oscillations before it is greatly damped down, it would be expected that the surface-layer of a uniformly magnetized steel needle would be either completely demagnetized or show evidence of several oscillations in opposite directions. The effect may be explained when the demagnetizing force of the ends of a needle on itself is taken into account. The first half-oscillation that tends to demagnetize the needle has the demagnetizing force of the ends of the needle assisting it, while the return oscillation has it in opposition. The return oscillation will not therefore be able to remagnetize entirely the surface-layer already affected, but a thin layer will be left in the interior; this layer is added to with every oscillation, until the final effect will be that the surface of the needle will be magnetized in the opposite direction to the interior. The shorter the needle, the greater is the demagnetizing force of the ends upon it. The length found by experiments with a number of needles of different lengths to be most suitable for the conditions of the investigation was about $1 \frac{1}{2}$ centim.

A most important point to be considered is the thickness of the wire. The detector did not consist of a single wire, but of several of the same length made up in the form of a compound magnet, and the several wires insulated from one another by paraffin-wax to prevent eddy-currents. With a rapidly alternating current, each effect lasts for so short a time that the needle does not become demagnetized to any depth, and the outer layer then acts as a metallic screen for the interior. In such a rapidly alternating field as the leyden-jar circuit under consideration, where the alternations are of the order $10^{6}$ per second, the demagnetization is confined to a very thin layer on the surface. 'Thick wires are affected to a less depth than thin ones. Erskine (Wied. Ann. vol. lxii. 1897) has found that the screening effect on the needle in the solenoid B, where the first, third, and fifth half-oscillations demagnetize the needle, is less than on that in $A$, where the even oscillations are in opposition to the original magneti- 
zation; therefore the ratio $\gamma_{2} / \gamma_{1}$, i. e. the damping, will appear greater than it really is. The effect decreases steadily with the decrease in the diameter of the wire, and with very fine wires it is almost negligible. The detector used in all the experiments was composed of 55 hard steel wires $\cdot 0015 \mathrm{~cm}$. in diameter, very carefully insulated and made up into a compound magnet $1.5 \mathrm{~cm}$. in length. With such a needle, in a circuit where from theoretical considerations the damping could be shown to be very small, a damping of about 2 per cent. could be measured, so that any correction due to magnetic shielding in such a case must have been trifling.

The following arrangement, which is theoretically equivalent to that of the two solenoids described above, was adopted in practice.

A strip of brass was taken and bent into an almost complete circle (see fig. 1) which was fixed on a block of ebonite. At the centre of the circle an ebonite tube projected which served

Fig. 1.

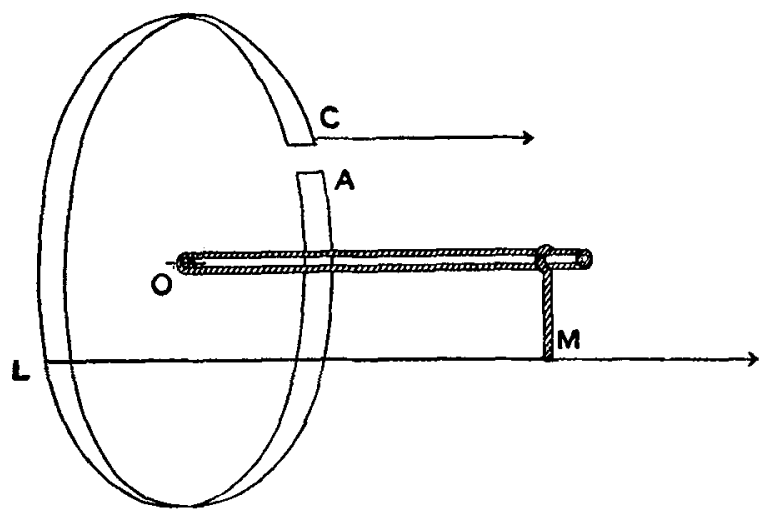

as the axis of a metal arm which pressed against the circumference of the circle, and could be moved about it. The detector was fixed in the end of a glass tube which could be easily slipped in and out of the central ebonite tube. A scale dividing the circle into 120 divisions was placed about its circumference, and the whole fixed in position before a mirrormagnetometer. The circle was placed in series with the discharge-circuit, one wire being connected to one extremity of the circle and the other to the movable arm, so that any desired portion of the circumference might be included in the circuit (fig. 1). This arrangement is practically a form of the tangent-galvanometer with a single coil, the intensity of 
the current being measured by the deflexion of the magnetometer due to the demagnetization of the detector-needle, instead of by the direct deflexion of a magnet at its centre.

Fig. 2.

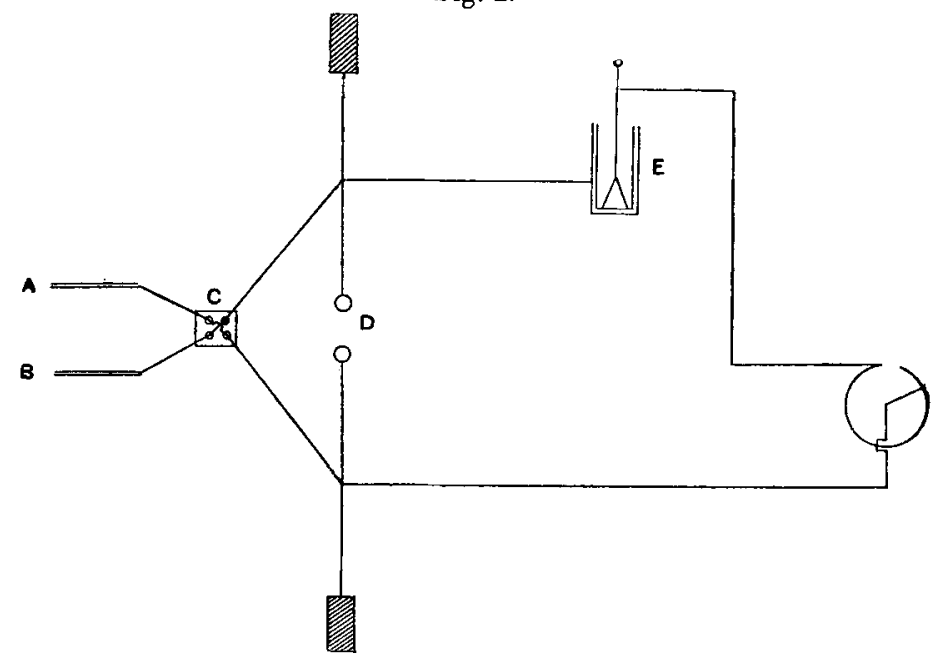

The discharge-circuit is shown in fig. 2. A and B are leads from the terminals of a large Wimshurst machine which supplied the current. $C$ is a key by means of which the direction of the discharge could be reversed; and at $D$ is an air-break whose terminals were brass balls $2 \cdot 1 \mathrm{cms}$. in diameter, and whose distance could be varied at will. E is the leyden-jar, to the outer coating of which a wire is led from one terminal of the air-break, while the other terminal is connected through the circle to the inner coating of the jar $\mathbf{E}$.

The magnetic field due to the wires leading out from the circle was found to affect the results. To prevent this the wires were carried out for some distance parallel to the needle and enclosed in a glass tube, which was in turn surrounded by a brass cylinder connected to earth, so that all magnetic effect due to this field was done away with. The remainder of the circuit was at too great a distance from the needle to have any effect on it. In this way it was ensured that the demagnetization of the needle was entirely due to the current through the arc of the brass circle. Since the magnetic field at the centre of a circle due to an are of length $l$ is given by $\mathrm{H}=\frac{l y}{r^{2}}$, where $\gamma$ is the current, we see 
that the magnetic force acting on the needle is proportional to the length of the are traversed by the discharge.

The circuit was rectangular in shape and measured 145 by $125 \mathrm{cms}$. The wires forming it were of copper $035 \mathrm{~cm}$. in radius, and the self-inductance of such a circuit calculated by the formula

$$
\mathrm{L}=4 \mu l \log _{e} \frac{b}{a},
$$

where $l=$ length of wires, $b=$ distance between them, and $a$ is their radius, was found to be about $10^{4}$ C.G.S. units. The capacity of the leyden-jar employed was 2500 E.S. units; therefore assuming $\mathrm{R}=0$, we get time of oscillation

$$
\begin{aligned}
\mathrm{T} & =2 \pi \sqrt{\mathrm{LC}} \\
& =\frac{2}{10^{6}}, \text { approximately; } \\
\therefore \quad \frac{\mathrm{T}}{2} & =\frac{1}{10^{6}} .
\end{aligned}
$$

If $\gamma_{1}, \gamma_{2}$ be the maximum currents for first and second halfoscillations respectively, then since the current at any time $\gamma$ is given by

$$
\gamma=\frac{\mathrm{C} \nu_{0}}{\sqrt{\sqrt{\mathrm{LC}}}} \cdot \epsilon^{-\frac{\mathrm{R}}{2 \mathrm{I}} t} \sin \frac{t}{\sqrt{\mathrm{LC}}} ;
$$

then

$$
\gamma_{1}=p \mathrm{C} v_{0} \epsilon^{-\frac{\mathrm{R}}{2} \mathrm{~L} \cdot \frac{\mathrm{T}}{4}}
$$

where $p=1 / \sqrt{\mathrm{LC}}$,

and

$$
\begin{gathered}
\gamma_{2}=p \mathrm{C} \nu_{0} \epsilon^{-\frac{\mathrm{R}}{2 \mathrm{~L}} \cdot \frac{3 \mathrm{~T}}{4}} \\
\therefore \quad \text { the damping } \gamma_{2} / \gamma_{1}=\epsilon^{-\frac{\mathrm{R}}{2 \mathrm{~L}} \cdot \frac{\mathrm{T}}{2}} .
\end{gathered}
$$

The observations were made in the following way:-The needle was magnetized to saturation in a solenoid which had a fixed iron core extending through part of its length. Against this core the needle was pressed so that it was thoroughly magnetized, and could be replaced in exactly the same position every time it was to be remagnetized. The needle was then placed in position at the centre of the circle, and the deflexion compensated by a neighbouring magnet. If the arm of the circle was at $\mathrm{C}$ (fig. 1), when a discharge passed no effect was produced on the needle; but if any part of the are was included there was a deflexion due to the partial demagnetization of the needle. The deflexion was noted, the needle removed, completely demagnetized, and

Phil. Mag. S. 6. Vol. 2. No. 7. July 1901. 
then magnetized again to exactly the same amount as before, and replaced in its position at the centre of the circle. The direction of the discharge was reversed, and the arm of the circle moved until the deflexions in the two cases were the same. When this is the case, the ratio of the maximum values of the first and second half-oscillations is given by the ratio of the arcs traversed by the discharge.

The amount of demagnetization of the needle is approximately proportional to the magnetic force measured by the length of the are of the circle through which the discharge passes until the needle is more than half-demagnetized, after which the magnetization falls off more slowly, and the eurve representing the relation becomes concave with respect to the axis along which the ares are measured (fig. 3,a). The circuit was always so arranged that the magnetic moment of the needle was not reduced to more than half its value by the passage of the discharge, and the measurements were thus always made on the straight part of the curve. When the current was very large, the part of the circuit containing the needle had to be shunted. Repeated experiments with and without the shunt when the current was just at its limiting value for use without it, showed that it made no difference in the final relations of the observations.

It was found rather troublesome in practice to adjust the arcs to give exactly the same deflexion for the two directions of the discharge, and a slight change was made in the method of making the observations which simplified them. The observations made in this way could be reduced by means of a calibration-curve to the exact equivalent of those obtained in the manner described before.

The needle was magnetized to saturation and placed at the centre of the circle as before, and the discharge passed in the direction in which the second half-oscillation demagnetized the needle; the deflexion was read, and then, without removing the needle or changing the arc of the circle, the discharge was reversed and the increased deflexion noted. By means of the curve (fig. 3. b) showing the relation between the are and the deflexion, the arcs corresponding to these deflexions were determined, and the damping found, as before, much more accurately than by the first method, where it was necessary to make a number of trials to find the exact are which would give the required deflexion.

The first discharge which passes through the circuit causes a reduction of the magnetization, the amount depending on the arc, the period of oscillation, and the resistance. A few 
successive discharges in the same direction slightly increase the amount, but the needle soon reaches a steady state so that the passage of any further number of discharges has no further effect. Care was taken to have a sufficient number of discharges in one direction to reduce the magnetization to a steady state before the direction of the current was changed, and the same number of discharges took place in the reversed direction.

Fig. 3.

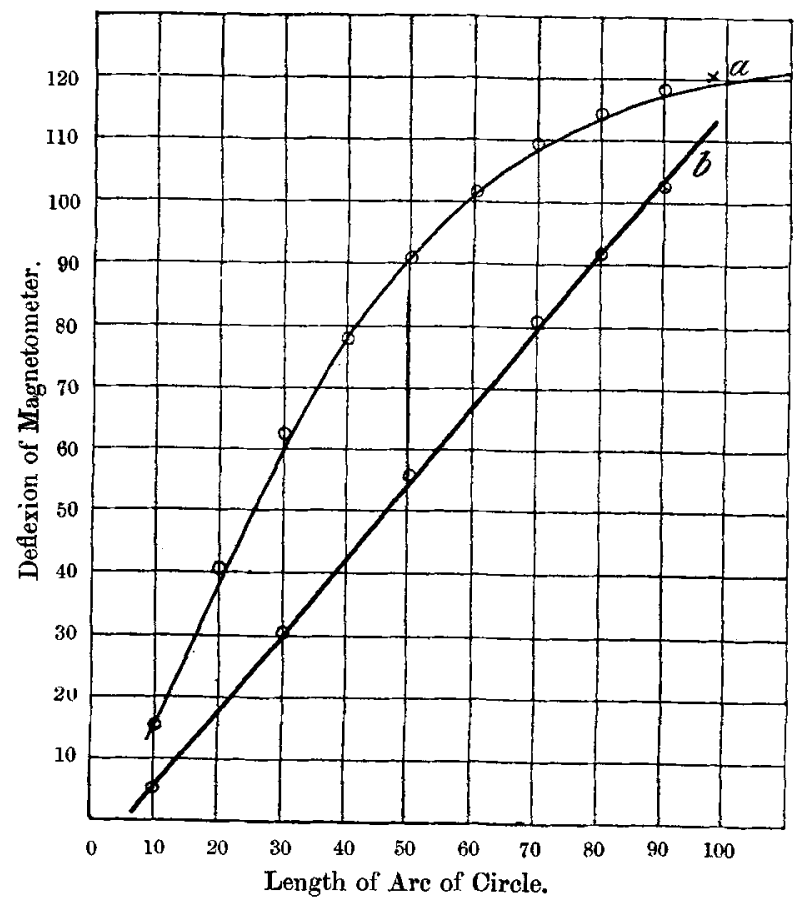

The damping increases steadily with the length of the spark, all the other conditions of the circuit remaining the same. The amount of this increase for a given range of spark-lengths was found to differ according to the amount of moisture in the atmosphere: the greater the moisture the more uniform was the damping. The followin? table gives the means of a large number of results on sparks varying from 1 to $13 \mathrm{~mm}$. in a moderately dry atmosphere.

H 2 


$\begin{array}{cl}\text { Spark-length. } & \gamma_{2} / \gamma_{1} \\ 1 \mathrm{~mm} . & .905 \\ 3 \% & .88 \\ 5 \% & .885 \\ 7 \% & .865 \\ 9 \% & -86 \\ 11 \% & .845 \\ 13 \% & .845\end{array}$

With smaller capacities the increase in the damping with increase of spark-length was much more rapid. The following values were obtained with a capacity of 725 C.G.S. units.

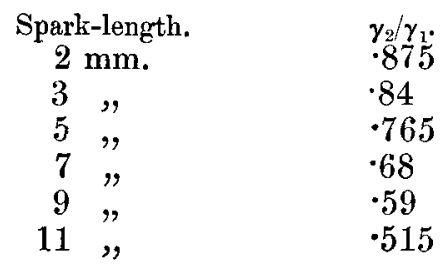

Fig. 4.

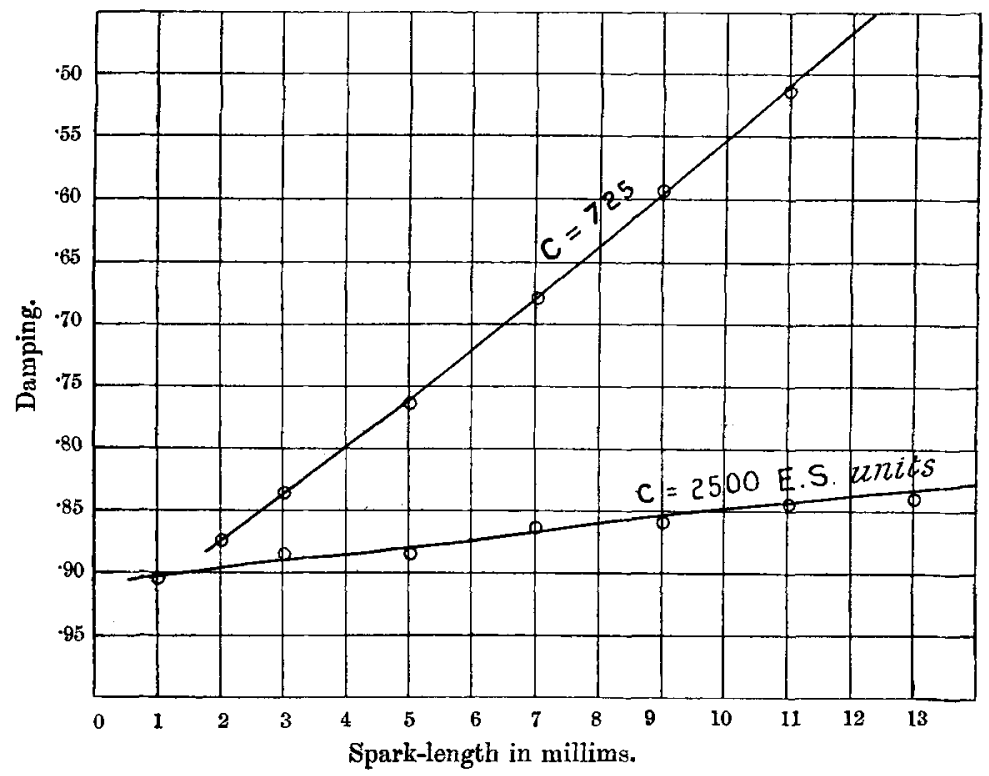

The curves showing the relation between the spark-length and damping in these two cases are given in fig. 4. 
A very small percentage of this damping is to be attributed to the resistance of the leads, for if $\mathrm{R}=$ resistance of the leads for an alternating current of frequency $10^{6}$ per second, then the damping due to this resistance is given by

$$
\gamma_{2} / \gamma_{1}=\epsilon^{-\frac{2 \mathrm{~L}}{\mathrm{R}} \cdot \frac{\mathrm{T}}{2}} .
$$

Now $\mathrm{R}=\sqrt{\frac{1}{2} \rho \mu l \mathrm{R}_{0}}$, where $l=$ length of wire in the circuit, and $R_{0}$ its resistance for direct currents. The circuit measured $125 \mathrm{cms}$. by $145 \mathrm{cms}$., therefore $l=540 \mathrm{cms}$, and diameter of wire $=\cdot 7 \mathrm{~mm}$.

Then

$$
\begin{aligned}
\mathrm{R} & =\sqrt{\frac{1}{2} \times 2 \pi \times 10^{6} \times 540 \times \frac{540 \times 1640}{\pi \times(035)^{2}}} \times \frac{1}{10^{9}} \mathrm{ohms} \\
& =58 \mathrm{ohm} .
\end{aligned}
$$

Then the damping due to $R=\epsilon^{-\frac{58}{2 \times 10^{4}} \times \frac{1}{2 \times 10^{06}} \times \frac{1}{10-9}}$

$$
\begin{aligned}
& =\boldsymbol{\epsilon}^{-\cdot 0145} \\
& =\cdot 985 \text { approximately, }
\end{aligned}
$$

which is a small damping. The damping in all the cases investigated is quite large compared with this, so that the expenditure of energy to which the damping is due must take place in the spark-gap. The dissipation of energy due to the excitation of electrical waves is very small in a leydenjar circuit, and may be neglected.

The ohmic resistance corresponding to the absorption of energy by the spark-gap was deduced by inserting in the circuit a known electrolytic resistance. If $R$ is the resistance of the air-break and leads, then since

then

$$
\begin{aligned}
& \gamma_{2} / \gamma_{1}=\epsilon^{-\frac{\mathrm{R}}{2 \mathrm{~L}} \cdot \frac{\mathrm{T}}{2}}=\rho_{1} \text { say, } \\
& \log \rho_{1}=-\frac{\mathrm{R}}{2 \mathrm{~L}} \cdot \frac{\mathrm{T}}{2}
\end{aligned}
$$

and if $R+r$ is the total resistance when the known resistance, consisting in the present instance of a solution of zinc sulphate with zinc electrodes, is inserted, the damping will be given by

$$
\begin{aligned}
& \rho_{2}=\epsilon^{-\frac{R+r}{2 L} \cdot \frac{T}{2}} \\
& \text { and } \quad \log \rho_{2}=-\frac{\mathrm{R}+r}{2 \mathrm{~L}} \cdot \frac{\mathrm{T}}{2} ; \\
& \text { then } \quad \frac{\log \rho_{2}}{\log \rho_{1}}=\frac{\mathbf{R}+r}{\mathrm{R}} \cdot \ldots \ldots \ldots \ldots \ldots
\end{aligned}
$$


$\mathrm{R}$ is therefore determined in terms of the known resistance. The value of $R$ determined from a number of observations in which different values of $r$ were used was about 2 ohms for a spark of $6 \mathrm{~mm}$. From this of course is to be subtracted the resistance of the leads, which is small in comparison with the total resistance.

When the resistance of the air-break is known, any unknown resistance $r_{1}$ can be determined in a similar manner ; for if $\rho_{1}$ and $\rho_{2}$ be the dampings without and with the unknown resistance respectively, then

$$
\frac{\log \rho_{2}}{\log \rho_{1}}=\frac{\mathrm{R}+r_{1}}{\mathbf{R}}
$$

and $r_{1}$ is therefore determined in terms of $R$.

This gives an accurate and practicable method of determining the values of resistances in rapidly alternating fields.

By inserting a series of increasing electrolytic resistances in the circuit, a curve showing the relation between the damping and the resistance was obtained. This experimental curve corresponded very closely with one found by calculating the values of the damping from the formula

$$
\rho=e^{-\frac{\mathbf{R}+\boldsymbol{r}_{0}}{2 \mathrm{~T}} \cdot \frac{\mathrm{T}}{2}}
$$

different values being substituted for $r_{0}$, and $\mathbf{R}$ being determined from one of the points on the experimental curve. The two curves are shown in fig. 5 .

The damping remained unchanged whatever the diameter of the copper wire employed in the circuit, the small increase of resistance with decrease of diameter was not sufficient to have any appreciable effect. When leads of iron wire, however, were substituted, the oscillations were greatly damped down, owing to the circular magnetization of the iron which prevents the current from sinking to any depth, and thus greatly increases the resistance. An iron wire $.7 \mathrm{~mm}$. in diameter gives a damping of 72 for a spark of $11 \mathrm{mms}$, while the damping for a copper wire of same diameter in the same circuit is $\cdot 85$. This increase of the damping for iron wires has been frequently noticed before, but by this method an exact measurement of the increase of resistance of an iron wire for any given rapidly alternating current can be obtained in a manner similar to that used to determine any other unknown resistance in the circuit. The damping was found to increase as the diameter of the iron wire decreased.

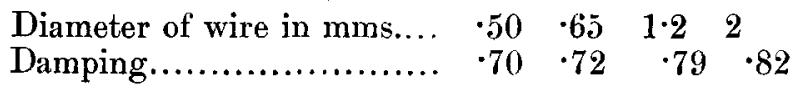


A very thin coating of copper deposited on the iron wire served to bring the damping back to its value for a copper wire.

Fig. 5.

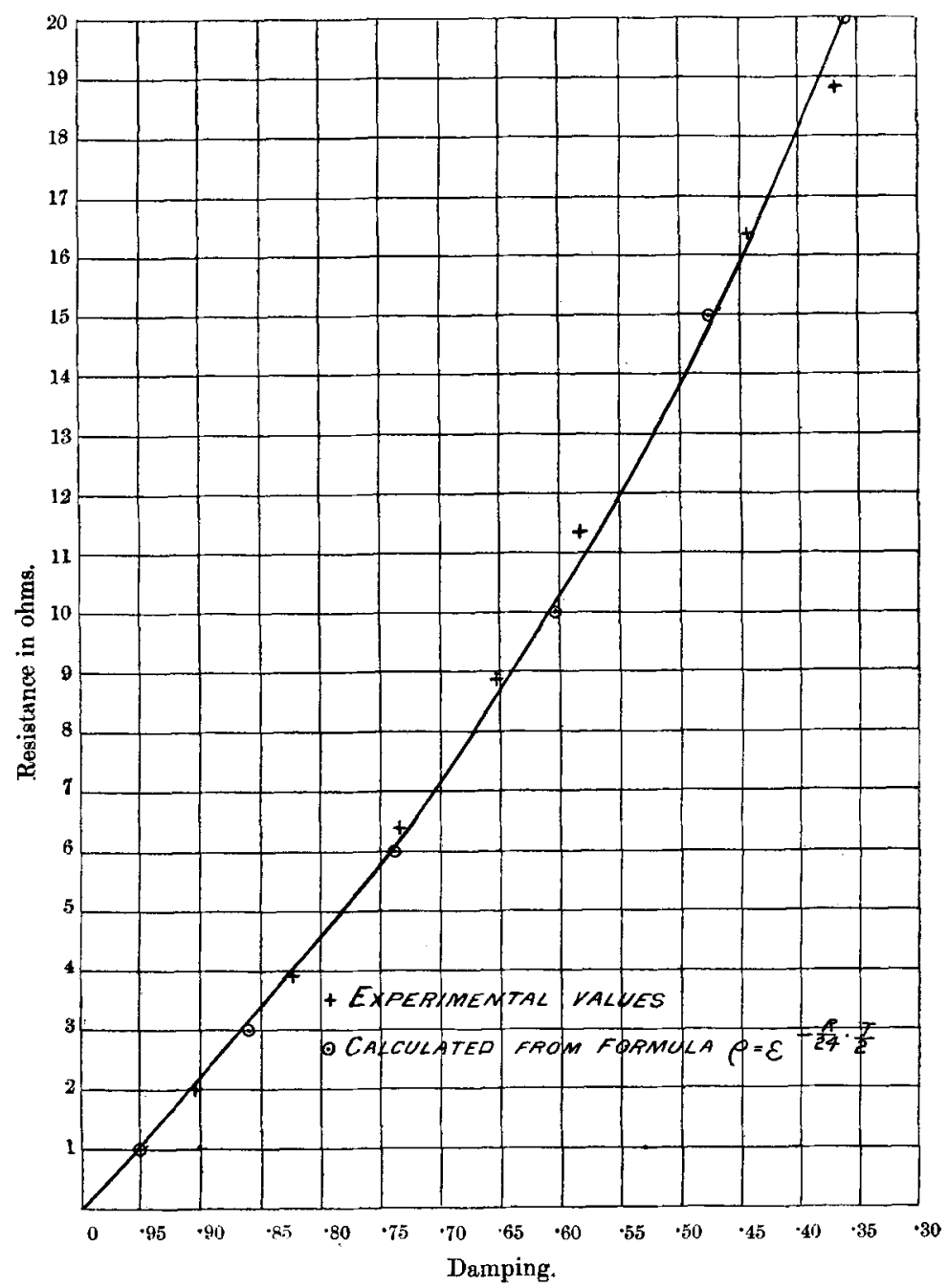

Instead of the brass balls at the air-break, balls made of iron and aluminium were substituted, but no difference in the damping conld be detected. The balls were always kept 
in a high state of polish, as the spark shows a disinclination to pass from any point on the ball which has become roughened or tarnished. It prefers to leap a longer distance from a smooth place.

To investigate the effect of increase of capacity on the damping, several jars were connected in parallel and placed in the circuit. The damping was found to be practically the same for one, two, four, eight, or twelve jars. The relation for smaller capacities than that of the leyden-jar employed in the first experiments, i.e., less than 2500 E.S. units, was obtained by means of an ebonite condenser whose capacity could be varied as desired by removing or adding to the tinfoil coating. It was found that the damping changed very slightly for capacities over 1000 C.G.S. units, but below that point the damping increased rapidly with decrease of capacity (see fig. 6).

This points to the same conclusion as that indicated by the curves for spark-length and damping with different capacities, that the damping reaches a comparatively steady condition when the current is large.

Change of pressure at the spark-gap had a very noticeable effect on the damping. The terminals of the air-break were enclosed in an air-tight glass bulb, which was connected to an air-pump. The air was thoroughly dried before entering the bulb.

The damping for any given spark-length decreases as the pressure becomes less. An exactly similar effect was observed when hydrogen and carbonic acid were introduced into the bulb instead of air. For a given spark-length and pressure the damping in hydrogen is less than in air. In fig. 7 is given a curve for pressure and damping with a spark of $1 \mathrm{~cm}$. in air, and also for a spark of $3 \mathrm{cms}$. in hydrogen.

Observations point to the existence of a critical pressure depending on the spark-length and the nature of the gas, below which the damping begins again to increase, evidently with great rapidity, for the resistance soon becomes so great that no discharge at all is obtainable. This point has not been very closely investigated, as the arrangement employed was not sensitive enough to be sufficiently affected by these very small currents. With a more sensitive arrangement accurate measurements might easily be made.

It has been shown that a very small part of the damping is due to the resistance of the leads, and in a leyden-jar circuit the loss of energy from radiation is also very small, so that by far the greater part of the dissipation of energy must take place at the spark-gap. 
Oseillations in the Discharge of a Leyden-jar.

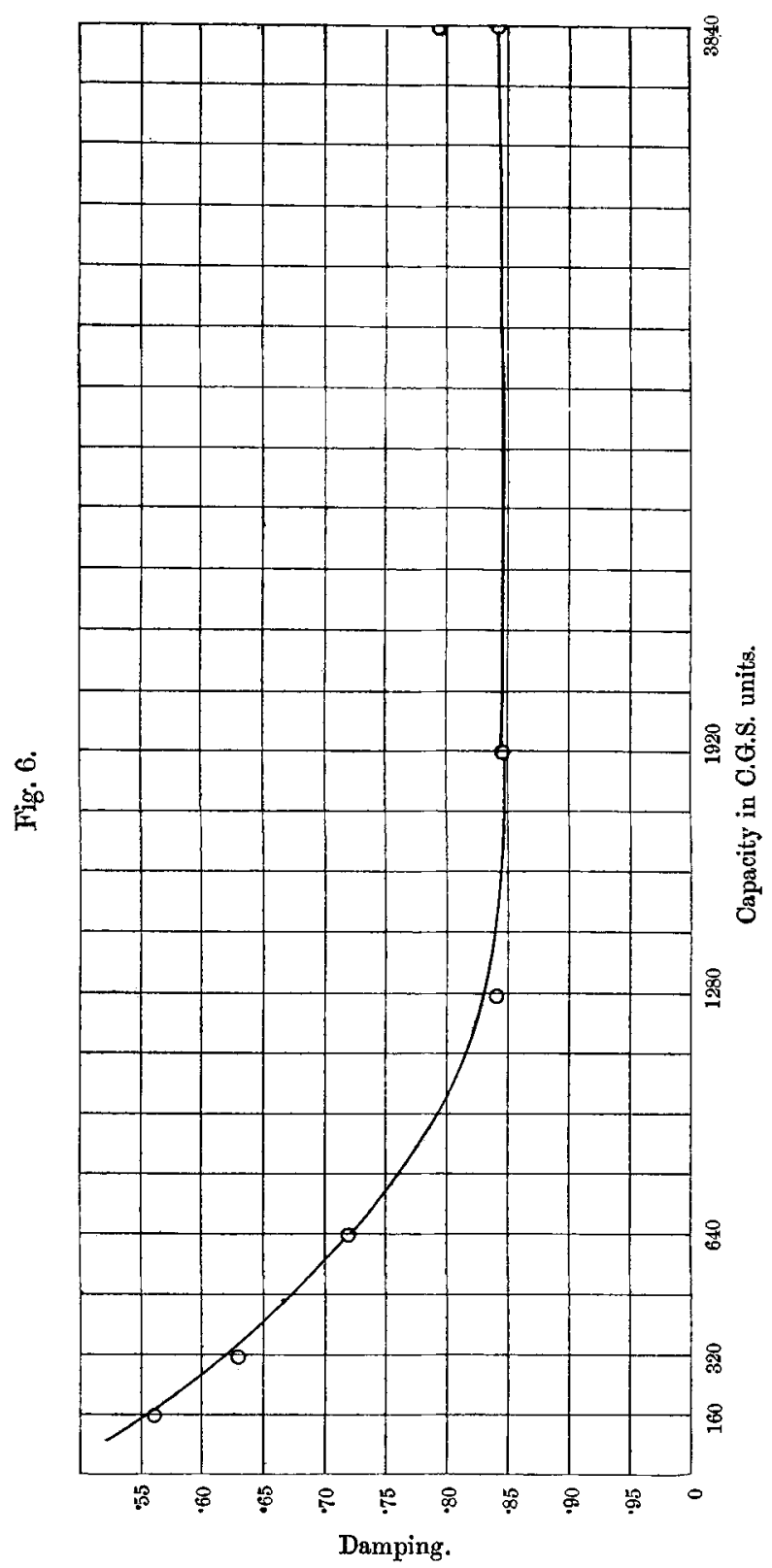


Consider the amount of energy required to dissociate the gas at the terminals into ions sufficient to carry across the currents. We will suppose that the ions are the same as are produced by $\mathrm{X}$-rays, and that the energy required to produce them is independent of the temperature. This will give a maximum expenditure of energy, since the energy required to produce the ions probably diminishes with rise of temperature.

Fig. 7.

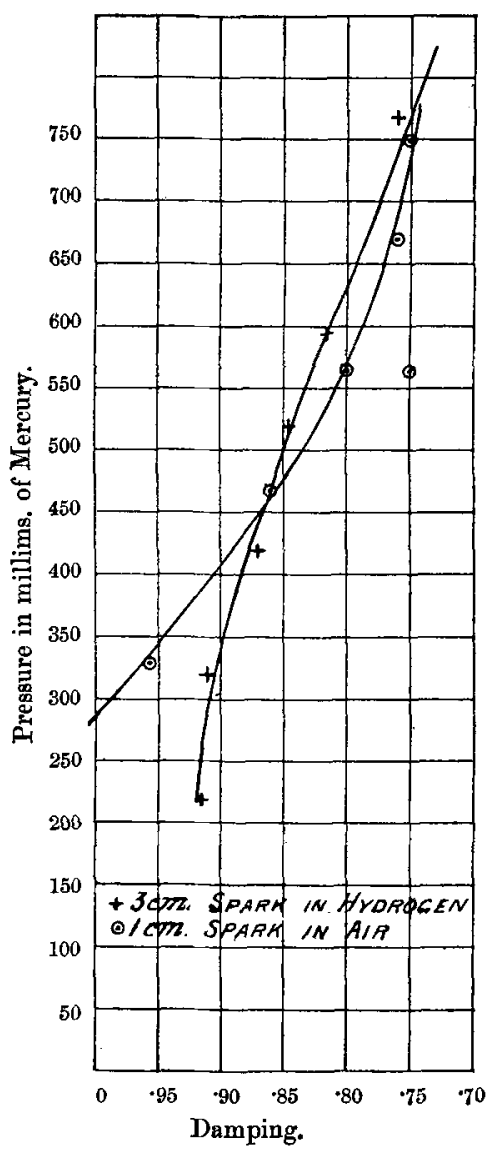

Maximum quantity of electricity conveyed over the airbreak in the first half-oscillation $=\mathrm{CV}_{0}$ approximately. 
Suppose spark-gap $=1 \mathrm{~cm}$.,

then $\quad V_{0}=10^{4} \times 4$ volts,

and $\quad \mathrm{C}=25 \times 10^{2}$ E.S. units for one jar ;

$$
\therefore \quad Q=\frac{25 \times 10^{2} \times 4 \times 10^{4}}{300}=\frac{1}{3} \times 10^{6} \text { E.S. units. }
$$

Now the charge on an ion has been found by J. J. Thomson to be equal to $6 \times 10^{-10} \mathrm{E}$.S. units, and the energy required to produce an ion (by $\mathrm{E}$. Rutherford and R. K. MeClung, Proc. Roy. Soc. vol. lxvii.) to be $=1.9 \times 10^{-10} \mathrm{ergs}$;

therefore energy required for the dissociation of 1 E.S. unit.

$$
=\frac{1.9 \times 10^{-10}}{6 \times 10^{-10}}=\cdot 3 \mathrm{erg}
$$

therefore energy required to carry across a quantity $Q$

$$
=\frac{1}{3} \times 10^{6} \times \frac{3}{10}=10^{5} \mathrm{ergs} .
$$

Now the total E.S. energy of the charge

therefore

$$
\begin{aligned}
& =\frac{1}{2} \mathrm{CV}_{0}^{2} \\
& =\frac{1}{2} \times 25 \times 10^{2} \times\left(\frac{4 \times 10^{4}}{3 \times 10^{2}}\right)^{2} \mathrm{ergs} \\
& =22 \times 10^{6} \mathrm{ergs.}
\end{aligned}
$$

Ratio of energy required to dissociate sufficient no. of ions

$$
=\frac{10^{5}}{22 \times 10^{6}}=\frac{1}{220}
$$

a damping of about 1-2 per cent.

If $V_{0}=10,000$, i.e. for a spark-gap of $\frac{1}{10}$ inch, the ratio is $1: 55$, a damping of about 2 per cent.

Thus we see that the expenditure of energy required to produce a sufficient number of ions to carry the discharge across is not enough to account for the damping. We are therefore driven to the conclusion that a much greater number of ions are produced than are sufficient to convey the discharge. The path of the discharge is very narrow and confined, and the ions so numerous and close together that recombination is very rapid. To these recombinations is 
probably due the high temperature of the spark. This high temperature in turn is favourable to the production of fresh ions, and to this fact the comparatively steady state of the damping in circuits with large capacities may probably be attributed. In such a case the current is so great that a large number of ions are produced, the recombination of these produce a high temperature by means of which fresh ions are formed to take the place of those that have recombined, and the field is thus kept comparatively uniform.

I wish to express my obligation and gratitude to Professor Rutherford for his kind assistance at every stage of the investigation.

Macdonald Physics Laboratory, McGill University. Feb. 12th, 1901 .

V. A Further Note on van der Waals' Equation. By Harold Hilton*.

TN a previous note $\dagger \mathrm{I}$ collected the various mathematical 1 facts connected with the family of curves obtained by considering the temperature a variable parameter in van der Waals' equation (the Isothermals); in the present note I propose to complete this work by doing the same for the curves of constant volume and the curves of constant pressure.

We take the same equation as before,

$$
3 y x^{3}-(y+8 \theta) x^{2}+9 x-3=0 . . . \quad \text {. . }
$$

We will first consider the family obtained by considering $x$ as an arbitrary parameter. The family is a series of straight lines such that two consecutire members meet in the point

$$
y=\frac{3 x-2}{x^{3}}, \quad \theta=\frac{(3 x-1)^{2}}{4 x^{3}} .
$$

Eliminating $x$ from these two ?equations, we have as the equation of the envelope

$$
(y+8 \theta-9)^{3}+81(y-4 \theta+3)^{2}=0,
$$

which has a cusp at the point $(1,1)$, the tangent to the cusp being $y-4 \theta+3=0$; cuts the axis of $\theta$ where $\theta=\frac{27}{32}$, and touches it where $\theta=0$; euts the axis of $y$ where $y=0$, and touches it where $y=-27$; and goes off to infinity in the direction of $y+8 \theta-9=0$. A series of points on it are given in Table $\mathrm{l}$. 